\title{
The Novel Integrating Sphere Type Near-Infrared Moisture Determination Instrument Based on LabVIEW
}

\author{
Yunliang Song ${ }^{1}$, Bin $\mathrm{Chen}^{2}$, Shushan Wang ${ }^{1}$, Daoli $\mathrm{Lu}^{2}$, and Min Yang ${ }^{2}$ \\ ${ }^{1}$ School of Mechanical Engineering \\ ${ }^{2}$ School of Food and Biological Engineering, \\ Jiangsu University, No. 301 Xuefu Road, Zhenjiang 212013, P.R. China \\ songyunliang@126.com, ncp@ujs.edn.cn
}

\begin{abstract}
Based on the technology of near-infrared spectroscopy, a novel nearinfrared moisture content determination instrument has been developed with three laser diodes of different wavelengths as light source, with one InGaAs detector as determination device, and with integrating sphere as sample holding device. Besides, LabVIEW virtual instrument program was employed for development of its operation instructions. This instrument has made full use of the integrating sphere system to remove the spectroscopic parts which are necessary in general spectrometers and has thus facilitated the improvement of system stability and signal noise ratio. Determination tests of moisture in sesame seeds and tea fresh leaves verified high precision of the instrument, showing that it can meet the primary requirement of moisture determination in practical production.
\end{abstract}

Keywords: NIR spectroscopy, moisture content, determination instrument, LabVIEW.

\section{Introduction}

Moisture widely exists in various natural substances. In medicine, food, tea, grain, tobacco, chemical and many other manufacturing industries, it's necessary to monitor the moisture content of raw materials and products in almost all aspects of processing. Hence, moisture measurement and control is an important aspect of the production, which directly affects product quality. Moisture content testing for wheat, corn, sesame seed, soybean, rapeseed and other agricultural products is directly related to product pricing and classification of seeds based on quality. The traditional methods of moisture content determination mainly drying method, resistance or capacitance measurement method and so on, are either testing process cumbersome, consuming too long time, or accuracy is not high enough, so cannot be used for non-contact measurement. Compared with these methods, Near-infrared (NIR) analysis technology has such advantages as non-destructive, fast, efficient, free of sample pretreatment, and suitable for process online analysis.

NIR is electromagnetic waves whose wavelength range within $780 \sim 2526 \mathrm{~nm}$, the earliest recognized waves in the non-visible light region. And after first used to 
determine water content in grains, NIR spectroscopy was subsequently used in quality assessment and analysis of other agricultural products. It is one of the earliest used and most effective ways for moisture detection in agricultural materials. In recent years, the development and production of NIR analyzer has progressed substantially in China. However, the existing NIR instruments generally use halogen lamps as light sources, which need to install splitting device, thus making the path of rays constitution and the control circuit system complex, the instrument debugging process tedious, the construction cost high, and practical application of the instruments difficult. In addition, they generally lack such functions as remote diagnosis, and remote data real-time transmission and processing. Furthermore, they have certain technical short comings as poor instrumental consistency between various parameters and high model transfer error.

To solve these problems and to promote the application of NIR special instruments, a novel NIR moisture determination instrument has been developed with integrating sphere as sample holding device, with three laser diodes (LD) of different wavelengths as light source, with an InGaAs detector, and with operation instructions developed using LabVIEW virtual instrument program. Rapid and non-destructive determination of moisture content of sesame seeds and tea fresh leaves have been completed on it.

\section{Overall Scheme Design}

The test system consists of two parts: hardware and software. Hardware is mainly used for standard optical signals generating and sending, reflected signal with sample information receiving, work process controlling and status monitoring. Software includes control and monitor interface, data analysis, and results displaying, developed with high-level graphical programming language LabVIEW.

Schematic and structure diagram of the integrating sphere type NIR moisture determination instrument are shown in Fig. 1 and Fig. 2. From the diagrams, a personal computer (PC) has been used to control the rotation interval of the 3 LD light sources inlayed in the integral ball along with InGaAs detector. The sample cell has been inserted into integral ball so as to eliminate measurement errors caused by the sample structural differences and realize body absorption, which makes full use of the structural characteristics of integrating sphere and good monochromaticity of LD to remove the spectroscopic parts and thus makes the device compact and stable. LD driven by match electric circuit is selected by the multiway switches to send out stable monochromatic

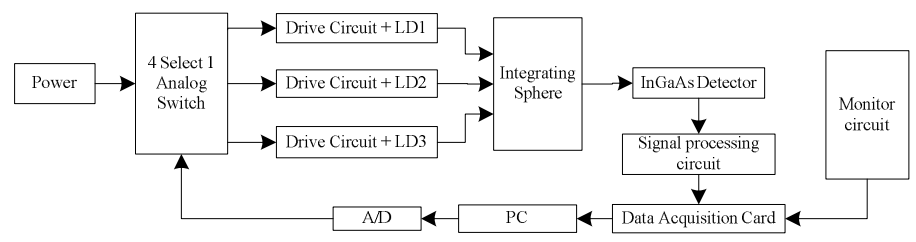

Fig. 1. Schematic of the integrating sphere type NIR moisture determination instrument 


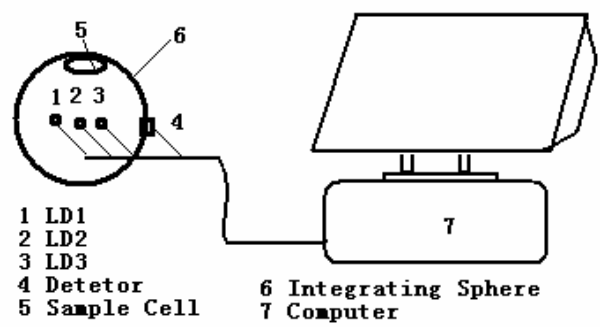

Fig. 2. Structure diagram of the integrating sphere type NIR moisture determination instrument

light. The reflection light signal with material function is then collected by the integral ball and transformed to the InGaAs detector. Through the data processing circuit, the signal enters the data acquisition card and then to the computer to run following processing to obtain the moisture content of the tested sample.

\section{Hardware System Design}

Hardware of the NIR moisture content determination instrument mainly consists of optical system and circuit system. As shown in Fig. 2, integrating sphere is the key component of the optical system. The integrating sphere is often used as a test sample devices in NIR test equipment, because it (1) can collect most of the analysis light, greatly increasing the signal strength, improving signal to noise ratio; (2) can eliminate the interference of reflection, scattering, polarization light, reducing the light incident impact of the shape and angle changes, making the instrument more stable and more reliable; (3) can reduce the impact on the measurement of uneven and spatial location as the sample changes, improving the measurement repeatability.

However, traditionally the sample room was located outside the integrating sphere, the light spot size and intensity of optical signal greatly affected the results. As the average moisture content is always the goal of moisture content determination, the sample room can be inserted into the integrating sphere, which is not only able to take full advantage of the benefits, but also increased the light intensity, thus make the influence subject to the measurement spot size disappear. The circuit system mainly includes light source driving circuit, detector detection and processing circuit, operation control and status monitoring circuit. And the light source driving circuit is essential to ensure the instrument precision from the wavelength and power stability of the emitted light.

\subsection{Optical System Design}

Integrating sphere is the key component of the optical system. To design it, not only the inner surface of the coating should have high reflectivity, and the integrating sphere opening area should also meet the general requirements of the integrating sphere. In this design, coating material for integrating sphere inner surface used gold to ensure its reflectivity of NIR up to $99.5 \%$. There are 5 openings in the integrating sphere, corresponding to the 3 light sources, a detector and a sample cell port. The 
diameter of the integrating sphere is $200 \mathrm{~mm}$. The different center wavelengths have been selected based on test data analysis of full spectrum instrument, namely 1310 $\mathrm{nm}, 1450 \mathrm{~nm}$ and $1550 \mathrm{~nm}$. Here, $1310 \mathrm{~nm}$ is used as reference wavelength, 1450 $\mathrm{nm}$ as water absorption peak, and $1550 \mathrm{~nm}$ as amended wave.

To eliminate temperature drift due to too long operation time, and revise operating point difference of the detector to receive signals from different light sources, the driving circuit was separately designed in view of various light sources to allow the light sources work in turn. In consideration of the semiconductor laser photo source's work principle and characteristic, one kind of simple feasible automatic power control (APC) driving circuit was used to realize the constant power control so as to remove the spectroscopic parts which are necessary in general spectrometers.

\subsection{Detection and Processing Circuit for Detector}

InGaAs photodiode sensitive in the long wavelength has been selected as detector. Yet its output current signal is very weak. Before being input to A/D transformation chip, there should be some processing circuits. As shown in Fig. 3, after the I/V transformation, chip DS1859 achieves the detector temperature control and filtering, amplifying processing, thus obtains voltage signal in the operating region of data acquisition card.

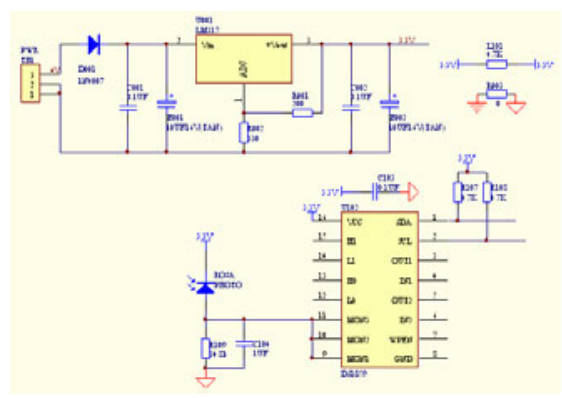

Fig. 3. Detector examination recuperation electric circuit

\subsection{Monitoring Circuit}

Equipment's work condition monitoring, includes environmental temperature testing, sample temperature testing, work status monitoring of light source and alarming. The current of the light sources are detected by Hall sensors, who can detect the current around the magnetic field without affecting work status of the instrument. And the temperature are detected by the digital temperature sensor DS18B20.

\subsection{Data Acquisition Card}

This system uses UA206D PCI bus A / D acquisition card sold by a company YouCai in Beijing. The card has two-way integrated D / A, with card-specific dynamic link library UA204.DLL, which provides simple and efficient acquisition and control 
functions to support various functions of UA206D. LabVIEW applications directly call Dynamic Link Library subroutines to realize a measurement process control and data acquisition.

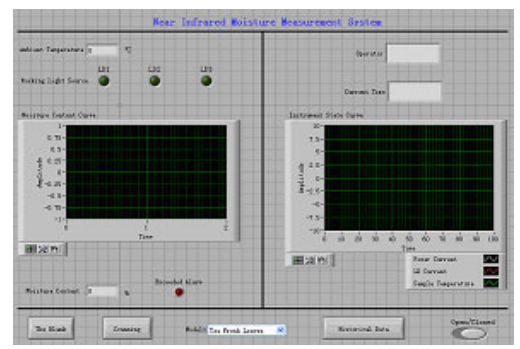

Fig. 4. Virtual instrument front panel

\section{Software System Design}

Software of the NIR moisture determination instrument mainly realizes instrument control, condition monitoring and data processing. Data processing includes data collection, data storage, the results display and processing. The man-machine interface is used to detect and determine the user input information to form the testing process and calls the corresponding subroutine. Background data processing fulfils analysis and judgments of the returned measurement data, forms statements and then archives. Software system controls apparatus of spectrum scanning to the measured sample while the background data processing is doing the analysis to determine whether the result reaches to the corresponding target.

As shown in Fig. 4, working process of the equipment contains: moisture content of continuous testing and monitoring, environmental temperature testing, sample temperature testing, work status monitoring of light source and alarming. The main interface will display the current operator status under the operator's rights and have different control responses on databases. During the test, the screen will display the progress of the main interface, show the data measured for some monitoring of the project as required in the interface, analyze data at the same time, and generate different forms of reporting results to the operator use. If requirements on sample are not satisfied, a warn message will appear.

\section{Moisture Content Test Experiments}

After assembly of hardware, design of software and debugging of the instrument, a test sample was scanned. Then, multi-dimensional linear regression (MLR) and polynomial regression algorithm was employed for establishing calibration model between relative value of system outputs and moisture content. Tea fresh leaf and sesame seed samples were used to carry out moisture content examination in the selected wavelength. The relative value of system outputs and moisture content has been established and survey examination has been carried out to the test sample. 
With a group of samples of known moisture content, first scan the blank on this instrument to define instrument operating point, and then load the samples and scan them to obtain system outputs in various wavelengths. Finally, set up the calibration model between relative value of system outputs and moisture content with software NIRSA2.2. System relative output is calculated as:

$$
H_{i}=I_{i} / I_{R}
$$

where, $H_{i}$ is the system relative output in detection wavelength $i, I_{R}$ is the reflected light intensity in reference wavelength $\mathrm{R}, I_{i}$ is the reflected light intensity in detection wavelength $i$. $(\mathrm{R}=1310 \mathrm{~nm}, i=1450 \mathrm{~nm}$ or $1550 \mathrm{~nm})$

\subsection{Moisture Content Modeling Analysis of Tea Fresh Leaves and Sesame Seeds}

The experiment altogether gathered 46 tea fresh leaf samples from the production line in a tea factory in Danyang, 30 samples as the adjustment collection, and 16 samples as the forecast collection. Scanning was carried out to examine separately to get the system relative outputs and actual moisture content of each sample, and water content measured according to GB / T 8304 -2002. 54 sesame seed samples were collected from the market in Zhenjiang, 36 samples as the adjustment collection, and 18 samples as the forecast collection. Water content was measured according to GB 5497-85, and was taken a similar testing process to the tea fresh leaves. Then the MLR model and the polynomial regression model were respectively established, and the model accuracy and error are shown in Table 1.

Table 1 shows that the integrating sphere type NIR water meter can meet the general moisture measurement requirements of the tea fresh leaves and sesame seeds. The model accuracy of tea fresh leaves is relatively poor, and the increase in precision from polynomial regression is also quite limited. This is mainly due to that the samples' loading tightness is difficult to control. As a result, the moisture content of tea fresh leaves spans a larger area, since working point adjustment device is difficult to achieve such a large measurement requirements. In sesame seeds modeling, the correlation coefficient of MLR model can reach above 0.90 . That of polynomial regression model can reach 0.97 , being sufficient to meet the accuracy requirement of the general test.

Table 1. Model precision of moisture measurement of tea fresh leaves and sesame seeds

\begin{tabular}{cllll}
\hline Model Precision & \multicolumn{2}{c}{ Tea Fresh Leaves } & \multicolumn{2}{l}{ Sesame Seeds } \\
\cline { 2 - 5 } & MLR & Polynomial & MLR & Polynomial \\
\hline $\begin{array}{c}\text { Correlation } \\
\text { Coefficient } \\
\text { of Model }\end{array}$ & 0.7339 & 0.7341 & 0.9251 & 0.9721 \\
$\begin{array}{c}\text { Correlation } \\
\text { Coefficient }\end{array}$ & 0.7318 & 0.7331 & 0.9175 & 0.9778 \\
of Predict & & & & \\
RMS Error & 0.0323 & 0.0323 & 0.0239 & 0.0148 \\
Ave. Error & $3.32 \%$ & $3.31 \%$ & $2.46 \%$ & $1.66 \%$ \\
Max. Error & $8.11 \%$ & $8.12 \%$ & $8.91 \%$ & $6.64 \%$ \\
Pre. MSE & $4.11 \%$ & $4.10 \%$ & $3.39 \%$ & $2.52 \%$ \\
\hline
\end{tabular}




\subsection{System Tests}

Stability. Without any conditions changed, collect the reflected light intensity 13 times in 5-minute interval. The results are shown in Fig. 5, and the error analysis are shown in Table 2.

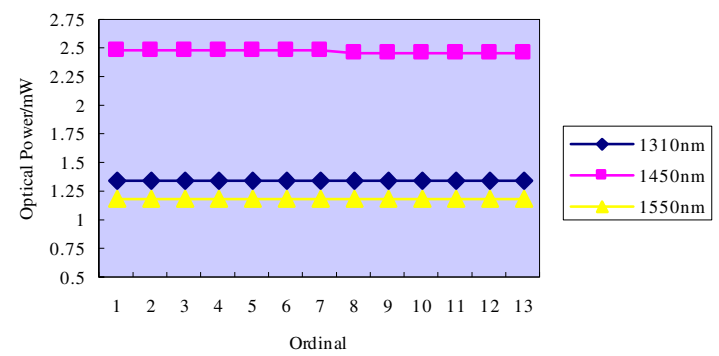

Fig. 5. Stability test results of the system

Table 2. Test result errors of stability (Unit: $\mathrm{mW}$ )

\begin{tabular}{lrrc}
\hline Error & \multicolumn{3}{c}{ Wavelength/nm } \\
\cline { 2 - 4 } & 1310 & 1450 & 1550 \\
\hline Max / Min & 1.347 & 2.470 & 1.174 \\
& $7 / 1.3457$ & $1 / 2.4638$ & $4 / 1.1727$ \\
Extreme Difference & 0.0020 & 0.0036 & 0.0017 \\
Mean & 1.3470 & 2.4666 & 1.1735 \\
Standard Deviation & 0.0006 & 0.0018 & 0.0004 \\
\hline
\end{tabular}

Repeatability. Take 2 sesame seed samples, each sample surveys the blank first, then surveys continuously 10 times, and record system relative outputs, substitute into sesame seed moisture content examination model to calculate water content, to test the repeatability of instrument. The results are shown in Table 3.

Error Analysis. Stability and repeatability of the instrument were obtained under the same loading condition. Considering the results of the model of tea fresh leaves and sesame seeds, the conclusion comes to that the stability of the system is high, and the system could meet the requirements of the prediction accuracy basically though the repeatability is relatively poor. Because the once reflect light from the sample is directly irradiate the detector, and the error caused by the sample compactedness in different loading is received and amplified directly. Duplication sample loading can improve forecast accuracy effectively, but it will affect the test speed and ease of use of the instrument, and also bring difficult to the transformation of online detection. Therefore, an adjustment on the internal structure of sphere accordingly is expected. 
Table 3. Test results of repeatability (\%)

\begin{tabular}{lll}
\hline Serial Number & Sample 1 & Sample 2 \\
\hline 1 & 6.46 & 7.68 \\
2 & 6.29 & 7.89 \\
3 & 6.64 & 7.69 \\
4 & 6.45 & 7.76 \\
5 & 6.76 & 8.12 \\
6 & 6.47 & 7.67 \\
7 & 6.45 & 7.69 \\
8 & 6.75 & 7.76 \\
9 & 6.56 & 8.11 \\
10 & 6.47 & 7.67 \\
Max / Min & $6.76 / 6.29$ & $8.12 / 7.67$ \\
Extreme Difference & 0.47 & 0.45 \\
Mean & 6.53 & 7.80 \\
Standard Deviation & 0.15 & 0.18 \\
\hline
\end{tabular}

\section{Conclusions}

Based on the technology of NIR spectroscopy and virtual instrument technology, a new moisture meter used for online analysis is developed. The results of system tests and moisture content detection experiment of tea fresh leaves and sesame seeds show that the instrument can meet the primary requirement of moisture determination in practical production. New application method of integrating sphere has greatly increased the signal intensity, enhanced signal to noise ratio of the instrument and reduced the influence caused by the changes of shape and angle of the reflected light, thus made the instrument more stable and reliable, as well as reduced the impact on measuring resulted from the sample unevenness and spatial changes, and improved the measurement repeatability. In addition, this system uses the rich software resources of computer to make some hardware software-based. To simplify the apparatus structure and shorten the development cycle of the instrument, it takes full advantage of the compatibility between LabVIEW software and non-NI hardware products, mostly uses domestic data acquisition cards to reduce costs. Users could create forecast model of water for different materials according to various needs, which can be easily ported to different applications. Meanwhile, the applications on remote diagnostics for the equipment failure also have been taken into account when designing the instruments.

\section{References}

1. Liu, J.: Practical near infrared spectroscopy. Science Press, Beijing (2008)

2. Lu, W.: Modern Near Infrared Spectroscopy. China Petrochemical Press, Beijing (2000)

3. Yan, Y., Zhao, L., Han, D.: Basic and Applied Near Infrared Spectroscopy. Chinese Light Industry Press, Beijing (2005)

4. Xu, F.: Near Infrared Moisture Meter Research and Development. Tianjin University (2005) 
5. Wen, M., Ji, H.: Development of portable Led-based NIR integrity wheat component measuring apparatus. J. Spectroscopy and Spectral Analysis 24, 276-279 (2004) (in Chinese)

6. Xiang, X., Wen, Z., Long, Z.: Research on the near-infrared spectrometer system. J. Spectroscopy and Spectral Analysis 29, 2286-2290 (2009) (in Chinese)

7. Zhan, X., Lin, J., Zhou, Z.: The technique of acquiring weak signal in NIR spectrometer. J. Chinese Journal of Scientific Instrument 23, 29-31 (2002)

8. Liang, X., Ji, H.: The measuring system of NIR based on virtual instrument. J. Microcomputer Information 24, 100-101 (2008) (in Chinese) 